\title{
A fluorescence approach to investigate repartitioning of coalescing agents in acrylic polymer emulsions
}

\author{
Tanzeela N. Raja $\cdot$ Albert M. Brouwer $\cdot$ Tijs Nabuurs $\cdot$ Ronald Tennebroek
}

Received: 2 October 2011 /Revised: 7 December 2011 / Accepted: 9 December 2011 /Published online: 27 December 2011

(C) The Author(s) 2011. This article is published with open access at Springerlink.com

\begin{abstract}
Repartitioning of co-solvents between particles of latex emulsions was investigated by means of a fluorescence method based on the detection of the amount of co-solvent via the solvatochromic shift of the emission maximum of a fluorescent probe, copolymerized at a low concentration. Complete repartitioning of co-solvents between particles of latex materials with a low $T_{g}$ (ca. $25^{\circ} \mathrm{C}$ ) occurred within minutes. For a hydrophilic latex with a $T_{g}$ of $68^{\circ} \mathrm{C}$, equilibration was achieved within an hour. Repartitioning was faster for more hydrophobic co-solvents. For a hydrophobic latex of similar $T_{g}$, co-solvent repartitioning took place on the same time scale, but complete equilibration was not reached. Possibly, there is an additional slow component in the repartitioning, or the prolonged presence of co-solvent causes a structural change in the latex particles that affects the outcome of the experiment.
\end{abstract}

Keywords Acrylic polymer emulsions .

Fluorescence spectroscopy . Coalescing agents .

Repartitioning of coalescing agent

\section{Introduction}

Waterborne polymer emulsions have gained much attention due to their environmental and economical advantages over

T. N. Raja $\cdot$ A. M. Brouwer $(\square)$

University of Amsterdam,

P.O. Box 94157, 1090 GD, Amsterdam, The Netherlands

e-mail: a.m.brouwer@uva.nl

T. Nabuurs $\cdot$ R. Tennebroek

DSM Coating Resins,

Sluisweg 12,

5145 PE, Waalwijk, The Netherlands

e-mail: Tijs.Nabuurs@DSM.com solventborne polymer coatings. They find application in various areas such as paints, adhesives, paper, inks, textile, surgical accessories, etc. In order to obtain good film properties, excellent film formation is required. To achieve this, a certain amount of coalescent agent is often used, which ultimately evaporates from the coating. Extensive research into environmentally more benign waterborne coatings with reduced amounts of such volatile organic compounds (VOC) is in full progress [1-3].

Latex film formation is influenced by several factors including the polymer composition, particle size (distribution) and particle morphology, drying conditions, molecular weight, nature and amount of surfactants, plasticizers and pigments, etc. [4]. Co-solvents play a vital role in film formation, as they facilitate particle deformation and polymer interdiffusion in the last stages of film formation [5]. A simple experimental characteristic for the formation of a crack-free and homogeneous film is the minimum film formation temperature (MFFT). It is defined as the temperature above which a smooth and coherent film is formed. Above MFFT, continuous film formation occurs due to particle deformation and interdiffusion. If a film is cast below MFFT, however, a discontinuous cracky or even powdery film is obtained. MFFT and $T_{g}$ of a polymer are often related [6]. Both are influenced by the same features such as coalescing agents, polymer composition, molecular weight, latex particle size, etc. Increasing the co-solvent concentration results in an approximately linear reduction of $T_{g}[7,8]$.

The mechanism of latex film formation is a somewhat controversial issue because different authors describe its evolution in two, three, or even four stages. The three-stage mechanism, however, appears to be the preferred one in the recent literature. Keddie and Routh stated in their recent book [9] that latex film formation 
takes place in three stages, which can overlap in time. These stages are (1) drying, (2) particle deformation, and (3) interdiffusion.

The role of co-solvent starts soon after the evaporation of water from the wet latex film (stage 1). In the case of a water-soluble co-solvent, evaporation of water triggers its entrance into the particles where it lowers $T_{g}$ and enhances coalescence. The co-solvent should evaporate after film formation so that a high $T_{g}$ of the dry polymer film can be obtained. Properties of an ideal co-solvent include: moderate evaporation rate, hydrolytic stability, and compatibility with a wide range of polymers. A co-solvent should obviously evaporate more slowly than water in order to be able to plasticize the particles. Moderately water miscible cosolvents will soften the outer shell of particles already in the wet stage and will penetrate into polymer particles and soften them inside upon film formation.

Waterborne polyvinyl emulsions can be prepared via emulsion polymerization $[6,10]$, yielding emulsified polymer particles in the size range of $20-500 \mathrm{~nm}$ in diameter. Formulation of such polymer emulsions with coalescing agents is carried out prior to their application. The partitioning of a co-solvent over the aqueous and polymer fractions depends on its relative solubilities in these two compartments. Therefore, knowledge of the distribution coefficients is important. No direct studies on the partitioning of cosolvents in polymeric dispersions can be found in the literature. Toussaint and DeWilde [11] proposed a model to estimate the distribution coefficients of coalescing agents between polymer particles and aqueous phase of the latex. They calculated the distribution coefficients for different coalescing agents in different latices and compared them with experimental literature values. The agreement was mostly satisfactory. Another model proposed by Toussaint et al. aims at estimating the reduction of $T_{g}$ or MFFT on addition of co-solvent to the latex [8].

Some other studies investigated the role of co-solvents by employing electrochemical and transmission techniques. In one such study, electrodeposition behavior determined the influence of Texanol on the morphology of films of acrylic latices with three different $T_{g} \mathrm{~s}\left(\right.$ low $\left(-10^{\circ} \mathrm{C}\right)$, medium $\left(21^{\circ} \mathrm{C}\right)$, and high $\left.T_{g}\left(101{ }^{\circ} \mathrm{C}\right)\right)$ [12]. The influence of co-solvent was found to be very system specific and depended upon the glass transition temperature of the latex. In another study, the role of coalescing agents in the film formation process was investigated by means of a turbidity technique by Zohrehvand and te Nijenhuis [13, 14]. Texanol and ethylene glycol monobutyl ether were used as coalescing agents to investigate the influences of drying temperature, aging time, and aging temperature on film formation $[14,15]$. Lahtinen et al. [16] described the synthesis of five epoxide-based reactive coalescing agents, which can react with the latex polymer and become part of the film, thus simultaneously increasing the solid content of the film as well as cross-linking it. Seebergh et al. [17] studied the effect of co-solvent concentration on the aggregation stability of a simple coating system.

Fu et al. [18] introduced a new strategy referred to as "Designed Diffusion Technology" which concurrently offers enhanced film formation with high mechanical properties and reduction in VOC of $\sim 30 \%$. Fu et al. used a two-polymer system in which a low $T_{g}$ (less than $\left.-5{ }^{\circ} \mathrm{C}\right)$ soft polymer in small amount $(15 \%$ to $35 \%)$ was added to a high $T_{g}\left(>20{ }^{\circ} \mathrm{C}\right)$ hydrophobic polymer, which made the dominant phase $(65 \%$ to $85 \%)$. Acrylic or styrene-acrylic polymer compositions were used as low $T_{g}$ soft polymer and Texanol as co-solvent. The selection of polymers was made such that the coalescing agent partitioned selectively into the high $T_{g}$ dominant phase polymer. During film formation, evaporation of water led to the repartitioning of the coalescing agent such that it preferred to migrate from the high $T_{g}$ polymer to the soft polymer. In the soft phase, the co-solvent diffused more rapidly leading to its faster evaporation from the film. Development of hardness was achieved at a faster rate without compromising mechanical properties. The authors did, however, not quantify the coalescent agent distribution.

Vignola et al. [19] observed that the effect of coalescing agent on a surfactant-stabilized latex varies with the nature of the coalescing agent. Paint flocculation was caused by the effect of the coalescing agent on the partitioning of surfactants between the polymer interface and the water phase. Extremely hydrophobic or hydrophilic coalescing agents have a tendency to significantly destabilize an emulsion and medium range hydrophobic solvents have less effect on the stability of the latex.

Fluorescence resonance energy transfer was recently used by Schroeder et al. to study the effect of co-solvents ethylene glycol (EG) and propylene glycol (PG) on polymer diffusion in poly(butylacrylate-co-methyl methacrylate) latex films [20]. They synthesized two similar emulsions except that one was labeled with the energy donor phenanthrene and the other with the acceptor aminobenzophenone. Latices were mixed in a 9:1 (acceptor/donor) ratio to monitor polymer diffusion at early stages of film formation. Schroeder et al. observed that both co-solvents retard polymer diffusion at early times of drying latex film but enhance polymer diffusion at later aging times. This was due to the partitioning behavior $\left(K=C_{\mathrm{W}} / C_{\mathrm{p}}\right)$ of both coalescing agents: at early drying time both $\mathrm{EG}$ and $\mathrm{PG}$ resided in the aqueous phase $(K>200)$ thus retarding coalescence and polymer diffusion. Once water evaporated and the film became dry, both coalescing agents partitioned in polymer phase thereby acting as plasticizers enhancing polymer particle interdiffusion.

Juhué and Lang used fluorescence energy transfer to monitor interdiffusion of latex particles [21]. Latices of poly $(n$ butylmethacrylate) (PBMA) labeled with phenanthrene 
(energy donor) and anthracene (energy acceptor) were drop cast on a quartz surface and annealed. From the phenanthrene fluorescence lifetime, the extent of particle interpenetration could be derived. The best film-forming agents were found to be those with a moderate evaporation rate. They found that increasing the Texanol concentration resulted in an increase of the fraction of mixing in the PBMA latex film. The diffusion constant increased with an increase in coalescing agent concentration and decreased with the increase in fraction of mixing [22].

From the above-mentioned examples from the literature, it is obvious that co-solvents have an enormous influence on latex film formation. They are an essential part of a latex coating to obtain a crack free, homogeneous film having the desired mechanical properties. We aimed to get a semiquantitative insight in the repartitioning behavior of co-solvents in polyvinyl polymer emulsions using fluorescence spectroscopy. A highly solvatochromic fluorescent probe (maleimidofluorotrope (MFT)) [23] covalently linked to the polymer backbone was employed for this purpose. The addition of a co-solvent to the latex leads to a shift of the emission of the probe to longer wavelengths, which is caused by the "softening" of the environment of the probe, as described elsewhere [24]. This method was applied in the present work to investigate the migration of co-solvent between latex particles in the wet state. We report the investigation of repartitioning of four co-solvents of different water solubility in hydrophobic and hydrophilic acrylic latices of low $\left(\sim 25^{\circ} \mathrm{C}\right)$ and high $\left(\sim 68^{\circ} \mathrm{C}\right)$ $T_{g}$. The water solubility of the four co-solvents employed in this study ranges from very low $(0.1 \%)$ to moderate $(24 \%)$. We will show that repartitioning is essentially complete within an hour for the high $T_{g}$ materials and much faster for the low $T_{g}$ latices.

\section{Experimental section}

\section{Materials}

The monomers styrene (Shell), 2-ethylhexylacrylate (FPC), methyl methacrylate (Proquigel) and ethyl acrylate (FPC) were used as received. The coalescing agent 2,2,4-trimethyl- 1,3-pentanediol mono(2-methyl propanoate) (Texanol, TEX) was obtained from Eastman. Di(propylene glycol) $n$-butyl ether (DPnB), ethylene glycol ethylhexyl ether (EEH), and ethylene glycol ethyl ether acetate (EEA) were obtained from Aldrich and were used as received. The fluorogenic probe molecule 1 was available from previous work in our laboratory [25]. Column chromatography of the crude compound $\mathbf{1}$ was carried out using silica gel as stationary phase and diethylether as the eluent. The retention factor $\left(R_{\mathrm{f}}\right)$ of 1 was 0.71 and the recovery was $93 \%$. Scheme 1 shows the structure and the co-polymerization of MFT.

Preparation of latex materials

The hydrophobic styrene-co-2-ethylhexyl acrylate co-polymers (S/EHA) and hydrophilic methyl methacrylate-co-ethyl acrylate $(\mathrm{MMA} / \mathrm{EA})$ co-polymer latices were prepared in batches in low $T_{g}\left(\sim 20^{\circ} \mathrm{C}\right)$ and high $T_{g}\left(\sim 60^{\circ} \mathrm{C}\right)$ versions by varying the ratio of "hard" to "soft" monomers. $T_{\mathrm{g}}$ values were predicted with the Fox equation (Eq. 1) [26].

$\frac{1}{T_{g}}=\sum_{n} \frac{X_{n}}{T_{g n}}$

In Eq. $1, X_{n}$ and $T_{g n}$ are the weight fractions and the glass transition temperatures (Kelvin) of the components in the corresponding homopolymers, respectively. All latices comprised $2 \% \mathrm{w} / \mathrm{w}$ acrylic acid, and it was used as a third monomer for the calculation of dry state $T_{g}$ of the final latex. $T_{\mathrm{g}} \mathrm{s}$ of the homopolymers polystyrene, poly(ethylhexyl acrylate), poly (methyl methacrylate), poly(ethyl acrylate), and poly(acrylic acid) were $100,-50,105,-24$, and $105^{\circ} \mathrm{C}$, respectively.

In the hydrophobic latex, the molar ratio of styrene ("hard monomer") and 2-ethyl hexylacrylate ("soft monomer") was 2.5:1 and 8:1, for low and high $T_{g}$, respectively. Hydrophilic latex materials with low and high $T_{g}$ were prepared by using different ratios of methyl methacrylate (hard) and ethyl acrylate (soft) monomers, namely $0.75: 1$ and $2.75: 1$ for low and high $T_{g}$ latices, respectively. Each latex was prepared in two batches, one without labeling and the other with labeling with copolymerized-1 (6 ppm).
Scheme 1 MFT (1) and its incorporation in a co-polymer

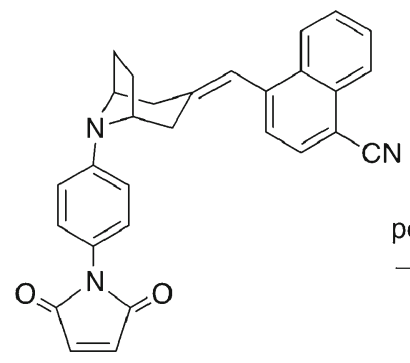

polymerization

MFT 1

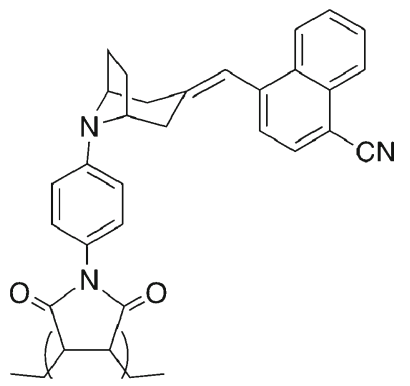

copolymerized MFT 
The actual $T_{g} \mathrm{~s}$ of the prepared latices were found to be a few degrees higher than the theoretical values of 20 and $60^{\circ} \mathrm{C}$. For low $T_{g}\left(20^{\circ} \mathrm{C}\right) \mathrm{S} / \mathrm{EHA}$ and MMA/EA latices, experimental values were 22 and $26^{\circ} \mathrm{C}$, respectively and high $T_{g}$ latices exhibited actual values of 69 and $68^{\circ} \mathrm{C}$, respectively. $T_{g} \mathrm{~s}$ of freshly prepared latex batches were determined using dry state differential scanning calorimetry. Freshly prepared latex was allowed to air dry for 2 weeks, and $T_{g}$ was measured without annealing or addition of a co-solvent.

Emulsion polymerization was carried out under $\mathrm{N}_{2}$ atmosphere in a 2-L reactor equipped with a mechanical stirrer with variable speed (100-1,000 rpm) and a reflux condenser. The monomer quantities for the different materials are given in Table 1. For labeling, compound $1(6 \mathrm{~mol} / \mathrm{mol} \mathrm{ppm}$ on monomers) was introduced via the monomer feed. The reactor was charged with water $(885 \mathrm{~mL})$ and sodium lauryl sulfate (SLS; $19.7 \mathrm{~g}, 1.3 \%)$. Ammonium persulfate $(1.8 \mathrm{~g} ; 0.12 \%$ $w / w)$ was used as initiator. Part of the monomer feed (5\%) was introduced at once to the solution of water and SLS in the reactor at $50{ }^{\circ} \mathrm{C}$, and $30 \%$ of initiator solution was added to the reactor at once at $70{ }^{\circ} \mathrm{C}$. Next, the temperature of the reactor was increased to $85^{\circ} \mathrm{C}$, and the remaining monomer and initiator feeds were added to the reactor over a period of $90 \mathrm{~min}$. After finishing the monomer and initiator feeds, the polymerization was allowed to continue for $30 \mathrm{~min}$. A residual monomer burn up treatment was carried out by adding iso-ascorbic acid $(0.6 \mathrm{~g})$ and tert-butyl hydroxy peroxide $(t$-BHPO; $0.8 \mathrm{~g})$. After $30 \mathrm{~min}$, the reaction mixture was cooled down from $85{ }^{\circ} \mathrm{C}$ to room temperature. Proxel ULTRA10 (Arch Biocides) was added (1.5 g; containing $10 \%$ of 1,2-benzisothiazolin-3-one dissolved with potassium hydroxide). Finally, the latex was filtered through a $75-\mu \mathrm{m}$ filter cloth and was stored in clean bottles. The solid contents of the final latex materials were $40 \% w / w$. A detailed characterization of the materials was described in an earlier publication [24]. It is important to emphasize that co-polymerization of the MFT fluorescent label was found to have no influence on the physical and chemical properties of these latices.

Table 1 Monomer quantities used for the preparation of set of four non-labeled latices of low and high $T_{\mathrm{g}} \mathrm{S} / \mathrm{EHA}$ and MMA/EA

\begin{tabular}{lcccc}
\hline $\begin{array}{l}\text { Component } \\
\text { weight }(\mathrm{g})\end{array}$ & S/EHA20 & S/EHA60 & $\begin{array}{l}\text { MMA/ } \\
\text { EA20 }\end{array}$ & $\begin{array}{l}\text { MMA/ } \\
\text { EA60 }\end{array}$ \\
\hline Acrylic acid & 11.8 & 11.8 & 11.8 & 11.8 \\
$\begin{array}{l}\text { Styrene } \\
\text { 2-ethylhexylacrylate }\end{array}$ & 339.1 & 473.5 & & \\
$\begin{array}{l}\text { Methyl methacrylate } \\
\text { Ethyl acrylate }\end{array}$ & & 105.8 & & \\
\end{tabular}

Another set of four latex batches was prepared with the addition of $0.012 \mathrm{~g}$ MFT (1) in order to obtain labeled latices

\section{Co-solvents and formulations}

The four co-solvents employed in this study were 2,2,4trimethyl-1,3-pentanediol(2-methylpropanoate) (Texanol, TEX), ethylene glycol ethyl hexylether (EEH), di(propylene glycol) $n$-butyl ether (DPnB) and ethylene glycol ethyl ether acetate (EEA) (Fig. 1). The water solubilities are $0.1 \%$ for TEX [27], 0.2\% for EEH [28], 5\% for DPnB [29], and 24\% for EEA [30].

Formulated latices were prepared with $9 \% w / w$ co-solvent. Prior to the addition of co-solvents, the $\mathrm{pH}$ of the latices was raised to 7-7.5 using ammonia. Next, the coalescing agent was added slowly with continuous stirring by a mechanical stirrer over $30 \mathrm{~min}$ to obtain a stable formulation.

\section{Co-solvent partitioning scheme}

The design of the co-solvent repartitioning experiments is schematically illustrated in Fig. 2. The emission maximum of the fluorescent label attached to the polymer shifts to longer wavelengths with increasing co-solvent concentration in the polymer particle. Thus, when we add a latex formulated with co-solvent to a fluorescently labeled one which does not contain co-solvent, the emission maximum will shift to a longer wavelength (bathochromic shift) as the co-solvent diffuses and its concentration in the labeled part of the emulsion increases. By monitoring the fluorescence as a function of time, we can follow the redistribution of cosolvent between the polymer particles in the mixture.

Conversely, when we dilute a fluorescently labeled formulated latex with one that does not contain co-solvent, the concentration of co-solvent in the labeled part of the mixture will decrease, and the fluorescence maximum will shift to the blue (hypsochromic shift). When we mix equal amounts of formulated and non-formulated latex, at equilibrium the co-solvent concentration in the final labeled latex should be half of the original value.

In order to perform this experiment, four samples of one kind of latex were used, referred to as "Emulsions I-IV". Two emulsions were mixed to get one blend and the fluorescence emission of the blend was measured as a function of time. The shift of the emission provided direct information about the
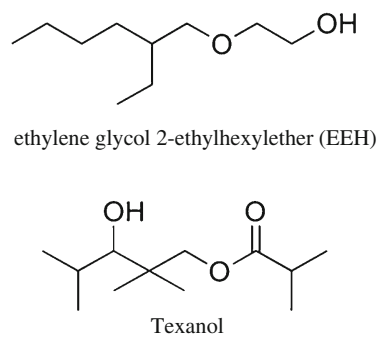

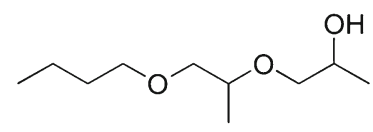

di(propylene glycol) $n$-butylether (DPnB)

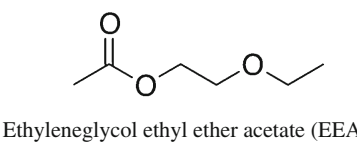

Fig. 1 Co-solvents used for co-solvent partitioning experiments 


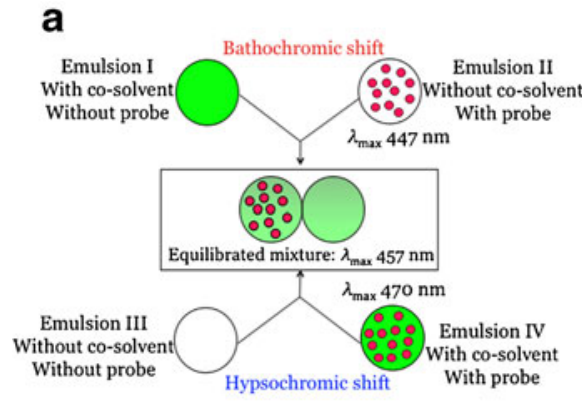

Fig. 2 a Schematic presentation of co-solvent repartitioning experiments; each big circle represents a latex particle of the respective emulsion. Emulsion I: green color represents co-solvent formulated latex particle which contains no dye molecules, Emulsion II: red circles depict co-polymerized dye molecules in the latex particle which

repartitioning of the co-solvent between the two types of polymer particles. Emulsion I was non-labeled and formulated, and Emulsion II was labeled but not formulated with cosolvent. Mixing these two yielded a blend whose emission was red-shifted compared to Emulsion II (bathochromic shift).

Emulsion III was non-labeled non-formulated, and when mixed with an equivalent amount of Emulsion IV, which was labeled and formulated, yielded a blend whose emission was blue-shifted, compared to the spectrum of Emulsion IV. This hypsochromic shift provided evidence of co-solvent leaving the labeled latex particles. Plotting hypsochromic and bathochromic shifts as a function of time, as shown in a hypothetical plot in Fig. 2b, provides information about the rate of repartitioning of the co-solvent. This approach was applied to all four sets of latices, i.e., low and high $T_{g}$, S/ $\mathrm{EHA}$, and MMA/EA for monitoring the repartitioning behavior of four different co-solvents.

\section{Blend formation}

Of each emulsion, $1 \mathrm{ml}$ (i.e., Emulsion I and II or Emulsion III and IV) was added into a quartz cuvet, and the whole amount was sucked once in and out of the pipette to ensure complete mixing of the two emulsions. Emission measurement was started within a minute after blending, and emission spectra were recorded for $1 \mathrm{~h}$. The acquisition of each spectrum (200 points, $0.5 \mathrm{~s} /$ point) took $100 \mathrm{~s}$. During this period, the spectrum may have changed due to the repartitioning process. However, as the results show that the dynamics of interest are either much faster or much slower than the acquisition time, the small distortion of the spectrum due to changes in the sample occurring during a scan can be neglected.

A trivial explanation of the observation reported below that some of the trend lines of the two complementary experiments did not meet could be that the amounts of the two emulsions that are blended are not well controlled. We carefully checked this by weighing the sample cell with one emulsion and again b

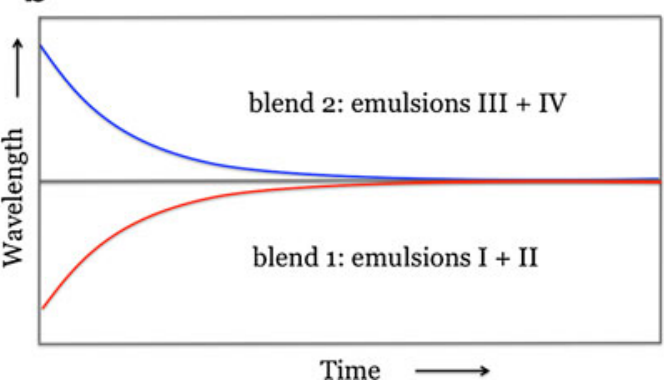

contains no co-solvent, Emulsion III contains neither dye nor cosolvent, and Emulsion IV contains both. b Expected trends of wavelength (emission maxima) versus time in the two complementary cosolvent repartitioning experiments

with the mixture. The error in the pipetting was $<0.5 \%$. Another possibility that we considered was that some heat could be generated during sample mixing or co-solvent repartitioning, which could affect the emission maximum [24]. Measurement of the temperature inside the blend during the experiment allowed us to exclude this as well. Each experiment was repeated three times to ensure good reproducibility.

\section{Results}

Low and high $T_{g}$ hydrophobic S/EHA and hydrophilic MMA/EA acrylic latices were separately formulated with four co-solvents, i.e., TEX, EEH, DPnB, and EEA according to the scheme illustrated in Fig. 2a. Co-solvent repartitioning was monitored using steady-state fluorescence spectroscopy. The emission measurements were started immediately after mixing equal amounts of the two latex emulsions in a quartz cuvet. For each experiment two blends were formed and emission spectra of each blend were measured for one hour during which 21 spectra were obtained. The time needed for each measurement was $100 \mathrm{~s}$. Fluorescence emission spectra were fitted with a skewed Gaussian function (Eq. 2) [31].

$I(\lambda)=I_{0}+I_{\max } \exp \left(-\ln 2\left[\ln \left(1+2 s\left(\lambda-\lambda_{\max }\right) w\right) / s\right]^{2}\right)$

In this equation, $I_{0}$ is a baseline parameter, $I_{\max }$ the maximum intensity, occurring at $\lambda_{\max }$. The width of the band is characterized by $w$, and the skewness by $s$. Higher skewness means more asymmetry in the curves, i.e., the tail on the long wavelength side will be longer, and the emission maximum will be located more towards the blue side of the curve.

The emission curves could be fitted well with the skewed Gaussian function. As an example, Fig. 3 displays the emission spectra of low $T_{g}$ S/EHA latex with $0 \%, 3 \%, 6 \%$, and $9 \%$ 


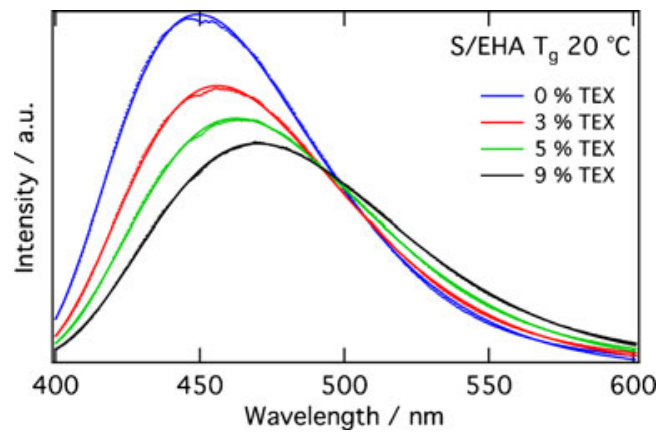

Fig. 3 Fluorescence emission spectra of low $T_{\mathrm{g}} \mathrm{S} / \mathrm{EHA}$ formulated with $0 \%, 3 \%, 6 \%$, and $9 \% w / w$ TEX. Emission spectra (dots) are fitted with a skewed Gaussian function (solid lines, according to Eq. 2), which yielded emission maxima, intensities, widths, and skewnesses listed in Table 2

$w / w$ TEX with the fitted function. The parameters obtained are listed in Table 2.

In this study, all emission spectra measured for monitoring the co-solvent repartitioning were similar in shape to those shown in Fig. 3. All of these curves could be fitted well with the skewed Gaussian function. Addition of co-solvent resulted in a shift of $\lambda_{\max }$ to longer wavelength. Emission intensity and skewness decreased and the width of the band increased. This trend was observed for all emission spectra measured in this study.

The precision of the data was checked in terms of error in emission measurement by the fluorescence spectrophotometer. In one test we measured the emission of an MFT model compound (described elsewhere) [24] in toluene at room temperature at various times of the day and on different days within a period of 2 weeks. Between measurements, the sample was taken out of the spectrometer, and the spectrometer was turned off overnight. This provided a measure of the error in the determination of the emission maximum of a representative sample: the standard deviation in $\lambda_{\max }$ was $\sim 0.5 \mathrm{~nm}$.

On the other hand, reproducibility of measurements of emission of co-polymerized MFT in latex is another issue because the result was found to depend on the age of the latex. Freshly prepared and more than 1-year-old latex

Table 2 Emission maximum, intensity, width, and skewness obtained by fitting the emission curves in Fig. 3 with the skewed Gaussian function (Eq. 2)

\begin{tabular}{lllll}
\hline $\begin{array}{l}\% / \\
w \text { TEX }\end{array}$ & $\begin{array}{l}\lambda_{\max } \\
(\mathrm{nm})\end{array}$ & $\begin{array}{l}\text { Intensity } \\
\text { (a.u.) }\end{array}$ & $\begin{array}{l}\text { Width } \\
(\mathrm{nm})\end{array}$ & Skewness \\
\hline 0 & 449.4 & $1.2 \times 10^{6}$ & 82.2 & 0.42 \\
3 & 456.1 & $9.3 \times 10^{5}$ & 88.4 & 0.39 \\
6 & 462.6 & $8.2 \times 10^{5}$ & 94.6 & 0.37 \\
9 & 469.9 & $7.3 \times 10^{5}$ & 100.4 & 0.36 \\
\hline
\end{tabular}

showed differences in emission maxima of up to $5 \mathrm{~nm}$, which is much larger than the measurement error of the spectrophotometer. Influence of temperature is another potentially significant factor to mention here; co-polymerized MFT in MMA/EA latex exhibited a 12-nm shift of the emission maximum towards longer wavelength upon increase in temperature from 15 to $35^{\circ} \mathrm{C}$ [24]. This shows that variation in (room) temperature will influence the position of the emission maximum of co-polymerized MFT. The temperature in the laboratory was mostly close to $23{ }^{\circ} \mathrm{C}$, but variations of $\pm 2{ }^{\circ} \mathrm{C}$ may have occurred. By inserting a thermocouple into a latex sample, we confirmed that temperature changes upon mixing of two latices and during the subsequent $1 \mathrm{~h}$ measurement were negligible.

Low $T_{g}$ hydrophilic latex

The low $T_{g}$ hydrophilic latex was composed of a co-polymer of MMA/EA and $2 \%$ of acrylic acid. For the detection of cosolvent repartitioning it was formulated with three different co-solvents: TEX, DPnB, and EEA.

As explained above, blend 1 was formed by mixing Emulsions I and II, which exhibited a bathochromic shift due to the migration of co-solvent into the fluorescently labeled particles. Mixing of Emulsions III and IV yielded blend 2 which showed a hypsochromic shift as a result of migration of co-solvent out of the fluorescently labeled latex particles. Figure 4 presents the fluorescence emission spectra of MMA/EA formulated with DPnB and EEA. With Texanol very similar results were obtained.

The emission maxima of the non-formulated Emulsion II and DPnB formulated Emulsion IV were observed at 476.3 and $496.1 \mathrm{~nm}$, respectively. Blends 1 and 2 showed the expected bathochromic and hypsochromic shifts, respectively. Within the time it took to prepare the blend and place the cell in the spectrometer the emission maxima coincided at the equilibration point, which was at $486 \mathrm{~nm}$, the average of $\lambda_{\max }$ of Emulsions II and IV.

The emission maxima of the spectra were determined by fitting with the skewed Gaussian function and plotted against time (Fig. 5). It is clear that migration of all three co-solvents in this low $T_{g}$ latex occurred very rapidly, i.e., within the first minute after blend formation.

Low $T_{g}$ hydrophobic latex

A low $T_{g}$ hydrophobic latex was composed of the copolymer S/EHA, containing $2 \%$ of AA. For the detection of co-solvent repartitioning, it was formulated with three different co-solvents: TEX, DPnB, and EEA. Emission spectra obtained for blend 1 and 2 for low $T_{g}$ S/EHA latex formulated with these co-solvents were similar to those shown in Fig. 4. In TEX-formulated S/EHA, low $T_{g}$ latex 
Fig. 4 Steady-state

fluorescence spectra of

MMA/EA $T_{\mathrm{g}} 20^{\circ} \mathrm{C}$ latex

formulated with a DPnB and

b EEA. Spectra of Emulsions II

and IV were measured once,

and for blends 1 and 2, a series

of 21 spectra were measured

during $1 \mathrm{~h}$ a

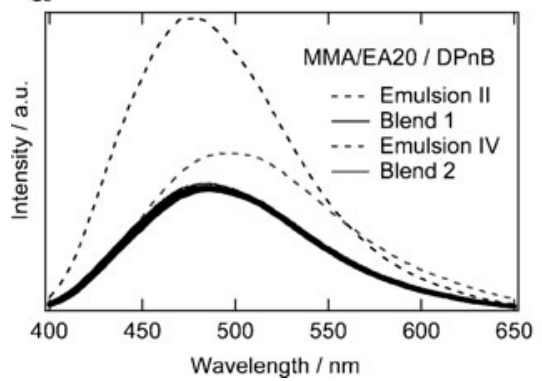

b

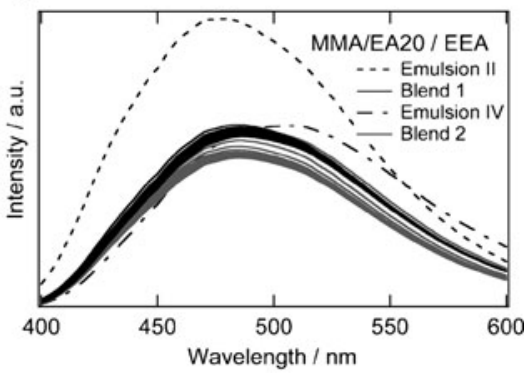

$\lambda_{\max }$ of Emulsion II and IV were observed at 449.4 and $470.0 \mathrm{~nm}$, respectively, and both blends exhibited $\lambda_{\max }$ near the average wavelength, i.e., $458 \mathrm{~nm}$. In DPnB-formulated $\mathrm{S} / \mathrm{EHA}, T_{g} 20{ }^{\circ} \mathrm{C}$ latex Emulsion II and IV were observed at 449.4 and $467.0 \mathrm{~nm}$, respectively. Blends 1 and 2 exhibited $\lambda_{\max }$ at 457.0 and $456.0 \mathrm{~nm}$, respectively, corresponding to $4-5 \%$ of co-solvent in the labeled fraction of the blend. The difference of $1 \mathrm{~nm}$ is not considered significant. Results of TEX closely resemble those of DPnB.

Figure 6 displays the fluorescence emission maxima versus time for the three co-solvent formulations of the low $T_{g}$ $\mathrm{S} / \mathrm{EHA}$ latex. Like the low $T_{g}$ MMA/EA latex, the formulations of S/EHA latices showed a rapid shift of the emission maxima of blends 1 and 2 to the equilibration point for TEX, DPnB, and EEA co-solvents. In the experiments with TEX and $\mathrm{DPnB}$, the spectra were monitored for $10 \mathrm{~min}$. The same experiment was done with EEA for $1 \mathrm{~h}$. It is clear that migration of all three co-solvents in this low $T_{g} \mathrm{~S} / \mathrm{EHA}$ latex occurred very rapidly, i.e., within the first minute after blend formation.
High $T_{g}$ hydrophilic latex

Co-solvent repartitioning experiments were carried out with a hydrophilic MMA/EA latex with high $T_{\mathrm{g}}\left(68^{\circ} \mathrm{C}\right)$. Emission maxima versus time plots of high $T_{\mathrm{g}} \mathrm{MMA} / \mathrm{EA}$ latex formulated with the four co-solvents, EEH, TEX, DPnB, and EEA, are shown in Fig. 7. For a quantitative description of the repartitioning of the co-solvents, we fitted the emission maximum versus time plots with exponential, biexponential, and stretched exponential functions. Stretched exponential functions did not show a reasonable fit for any of the plots, but simple mono and biexponential functions showed good fits. The data obtained are presented in Table 3. Two time constants resulted from biexponential fitting of the hypsochromic shifts for the emission versus time plots of EEH, TEX, and DPnB formulations. One time constant was large (9 to $14 \mathrm{~min}$ ) with a contribution of 1.7 to $2.9 \mathrm{~nm}$ while the other one was short (0.4 to $1.1 \mathrm{~min}$ ), with a larger contribution. Unlike these three co-solvents, EEA showed a good fit with the monoexponential model for
Fig. 5 Emission maximum versus time plotted for MMA/ EA $T_{\mathrm{g}} 20^{\circ} \mathrm{C}$ latex formulated with a TEX, b DPnB, and $\mathbf{c}$ EEA. The small differences in the emission maxima of the equilibrated blends are not considered significant
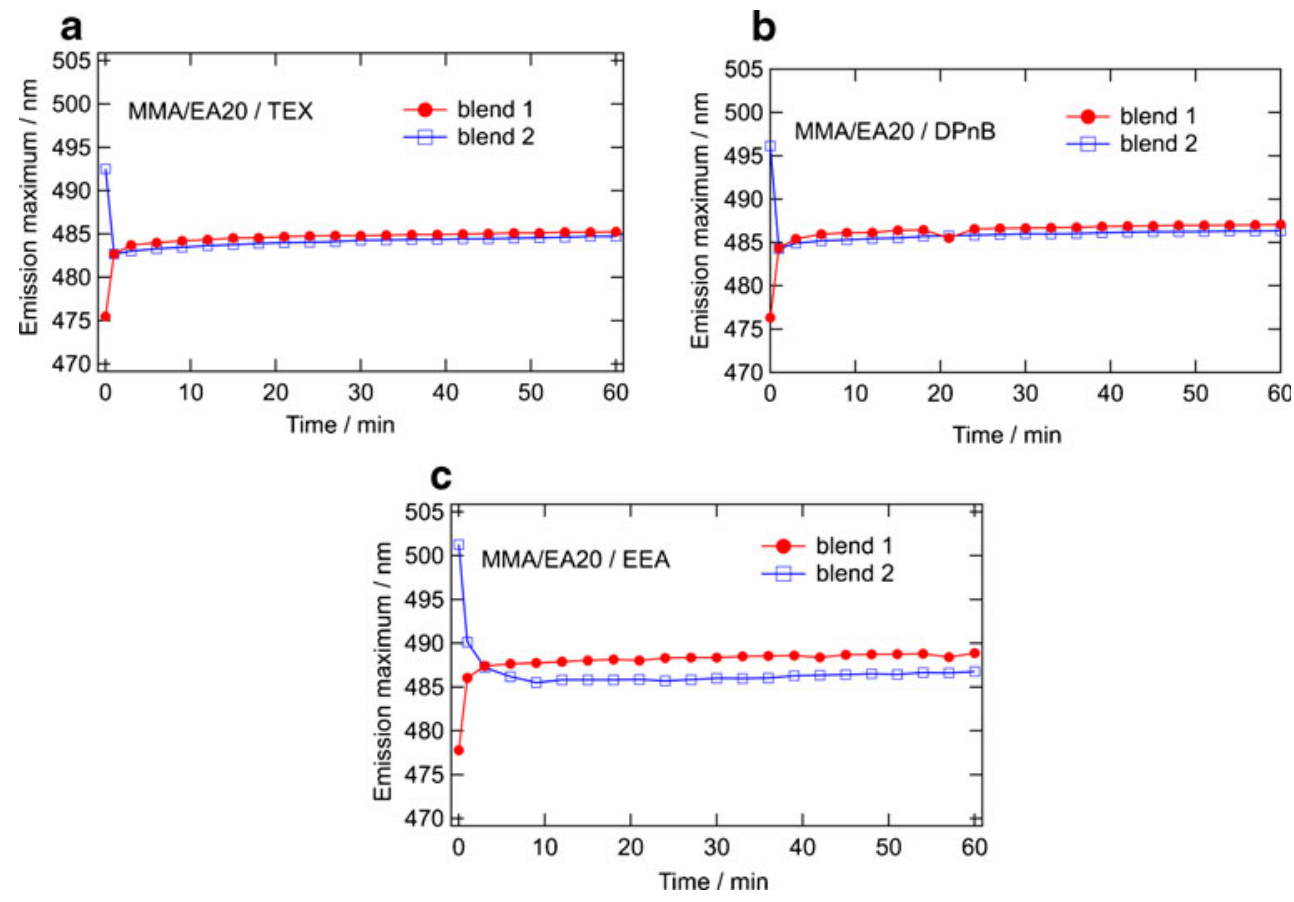
Fig. 6 Emission maximum versus time upon blending for S/EHA $T_{\mathrm{g}} 20^{\circ} \mathrm{C}$ latex formulated with a TEX, b $\mathrm{DPnB}$, and $\mathbf{c} \mathrm{EEA}$

\section{a}

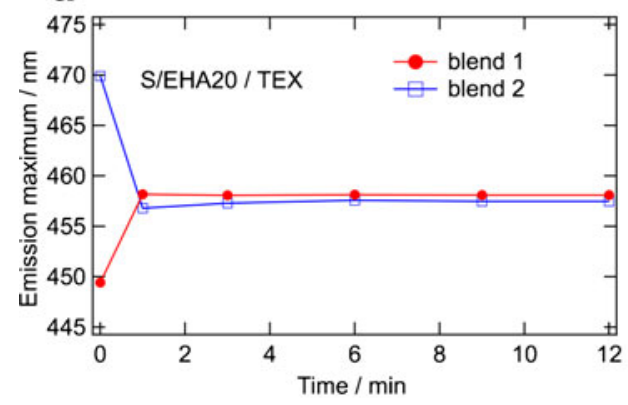

b

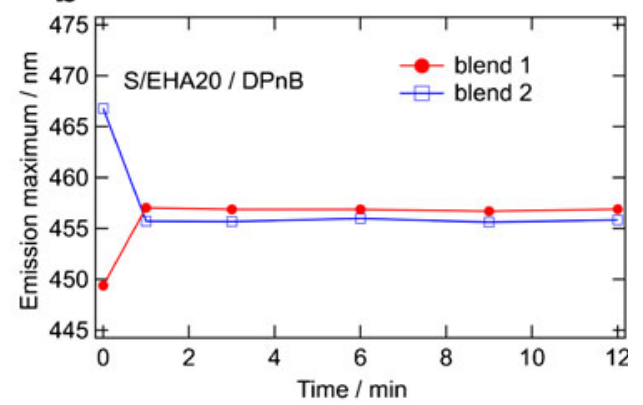

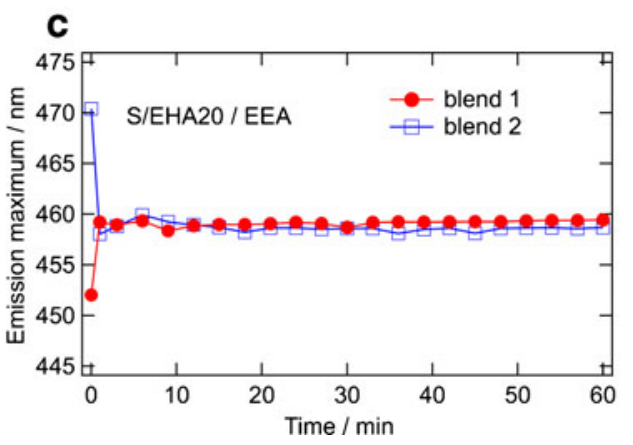

the hypsochromic shift, i.e., with a time constant of ca. $0.6 \mathrm{~min}$.

Bathochromic shifts for EEH and EEA co-solvents exhibited one time constant: 4.4 and $17 \mathrm{~min}$, respectively. For the bathochromic shift for TEX and DPnB formulations, a biexponential fit was slightly better than a monoexponential fit. The longer time constants are 21 and $12 \mathrm{~min}$, and the shorter ones are 3.7 and $3 \mathrm{~min}$, for TEX and DPnB, respectively. Because of the smaller amplitude of the slow component, the average time constant of the bathochromic shift is smaller for TEX (6 min) than for DPnB (8 min).

In the high $T_{g}$ hydrophilic latex, we observed a gradual shift of the emission maxima of blends 1 and 2 towards a common emission wavelength, which indicates an equilibration point in the migration of co-solvent into and out of latex particles. For EEH, equilibration was observed $20 \mathrm{~min}$ after blend formation, whereas for DPnB and TEX, it took about $40 \mathrm{~min}$. EEA took longer than the other three co-
Fig. 7 Emission maximum versus time plot for MMA/EA $T_{\mathrm{g}} 60^{\circ} \mathrm{C}$ latex formulated with a EEH, b TEX, $\mathbf{c} \mathrm{DPnB}$, and $\mathbf{d}$ EEA. Hypsochromic and bathochromic shifts in these plots were fitted with (bi) exponential functions, (solid lines)
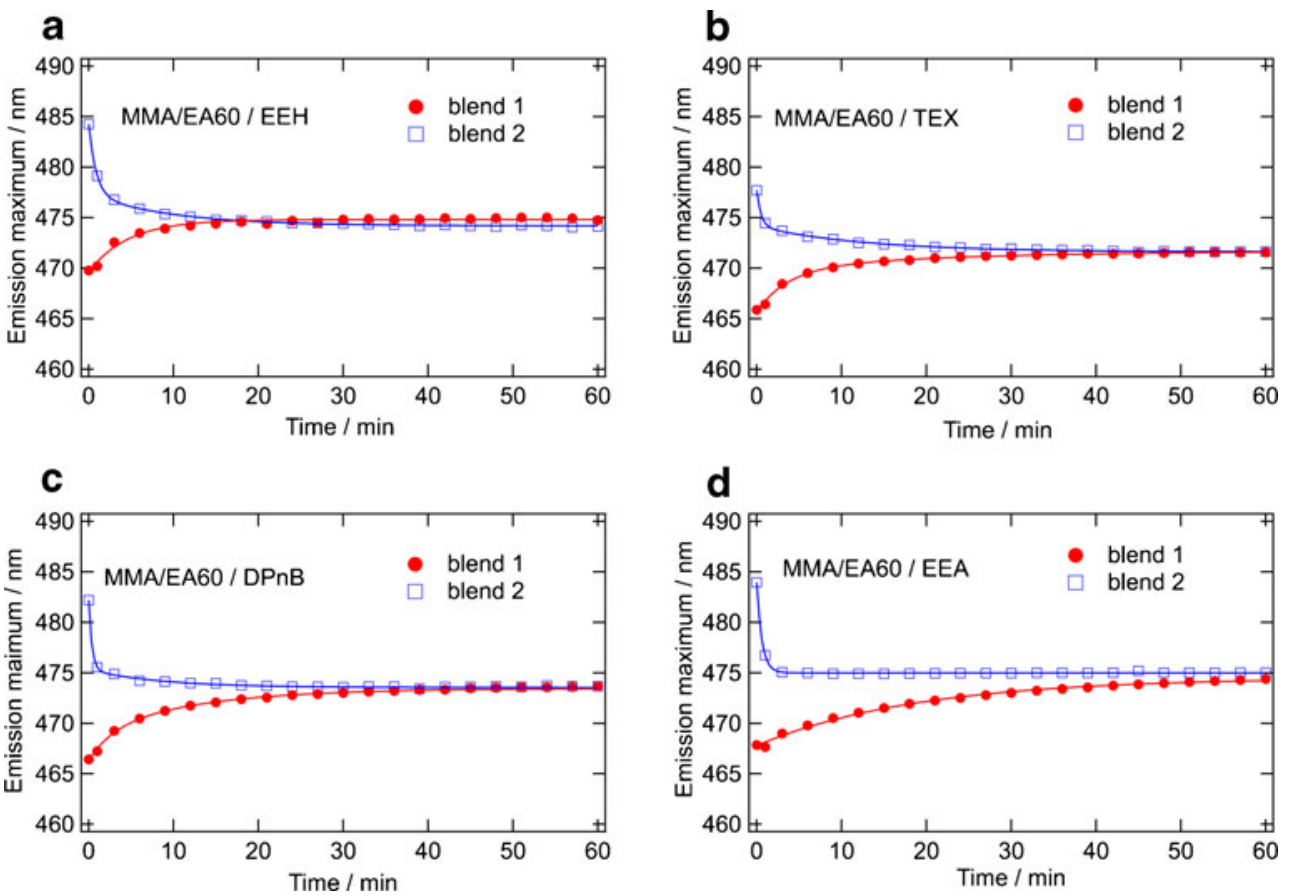
Table 3 Data obtained with (bi)exponential fitting of hypsochromic (blend 1) and bathochromic shifts (blend 2) in the emission maximum versus time plots of MMA/EA $T_{\mathrm{g}} 60{ }^{\circ} \mathrm{C}$ latex formulated with $\mathrm{EEH}$, TEX, DPnB, and EEA

\begin{tabular}{lcccc}
\hline $\begin{array}{l}\text { MMA/EA } T_{\mathrm{g}} 60 \\
{ }^{\circ} \mathrm{C} \text { formulation }\end{array}$ & $\tau_{1}(\mathrm{~min})$ & $A_{1}(\mathrm{~nm})$ & $\tau_{2}(\min )$ & $A_{2}(\mathrm{~nm})$ \\
\hline EEH-hypsochromic & $14 \pm 2$ & $2.9 \pm 0.4$ & $1.1 \pm 0.1$ & $7.4 \pm 0.4$ \\
EEH-bathochromic & $4.4 \pm 0.6$ & $5.1 \pm 0.3$ & & \\
TEX-hypsochromic & $13 \pm 1$ & $2.5 \pm 0.1$ & $0.51 \pm 0.04$ & $3.6 \pm 0.1$ \\
TEX-bathochromic & $21 \pm 6$ & $1.9 \pm 0.5$ & $3.7 \pm 0.5$ & $4.1 \pm 0.6$ \\
DPnB-hypsochromic & $9 \pm 2$ & $1.7 \pm 0.4$ & $0.4 \pm 0.1$ & $6.9 \pm 0.4$ \\
DPnB-bathochromic & $12 \pm 3$ & $4.4 \pm 1.6$ & $3 \pm 1$ & $2.8 \pm 1.7$ \\
EEA-hypsochromic & $0.6 \pm 0.1$ & $9.1 \pm 0.5$ & & \\
EEA-bathochromic & $17 \pm 1$ & $6.5 \pm 0.2$ & & \\
\hline
\end{tabular}

Fits are shown in Fig. 7

solvents, but the equilibration point was approximately reached after $60 \mathrm{~min}$.

High $T_{g}$ hydrophobic latex

Figure 8 presents the $\lambda_{\max }$ versus time plots for the co-solvent repartitioning experiments with high $T_{g} \mathrm{~S} / \mathrm{EHA}$ latex formulated with EEH, TEX, DPnB, and EEA. Fitting of the hypsochromic shifts in the case of TEX, DPnB, and EEA was not possible, but the EEH formulation showed a good monoexponential fit with a time constant of 6 min (Table 4). The bathochromic shifts showed a good fit with biexponential models. The time constants obtained by these fits describe the diffusion of co-solvent into the non-formulated latex particle.
In Fig. 8, two features are commonly observed in the plots for all four co-solvents: (1) coincidence of $\lambda_{\max }$ at a common equilibration point was not observed in the first hour of blend formation; the emission maxima remained 2 $3 \mathrm{~nm}$ apart, (2) escape of co-solvent was much more rapid and abrupt than the entrance, which progressed more gradually. However, no more change in $\lambda_{\max }$ was detected after approximately $40 \mathrm{~min}$ after blending. This was contrary to the hydrophobic latices of low $T_{g}$, which showed coincidence of hypsochromic and bathochromic shifts at the equilibration point in first minute of blend formation.

\section{Discussion}

An important result that emerges from our experiments is that the rate of co-solvent repartitioning is strongly dependent on the $T_{g}$ of the latex material, irrespective of the co-solvent. In low $T_{g}$ latices (dry state $T_{g}, 22-26^{\circ} \mathrm{C}$ ) repartitioning was found to be very rapid, whereas in high $T_{g}$ latices $\left(68-69^{\circ} \mathrm{C}\right)$ it was much slower. Still, it occurred within an hour for the high $T_{g}$ hydrophilic material. For the styrene-based copolymer of high $T_{g}$, the results for the two complementary measurements did not show convergence on the same emission wavelength, but most of the changes in the position of the emission maximum after blending occurred within an hour, as for the hydrophilic high $T_{g}$ material. These findings may be of practical importance for the coatings industry.

In the repartitioning process, the viscosity of the polymer particles appears to play a key role. In the low $T_{g}$ lattices, equilibration was observed in the first minute after mixing
Fig. 8 Co-solvent repartitioning experiment for $\mathrm{S} /$ EHA $T_{\mathrm{g}} 60^{\circ} \mathrm{C}$ latex formulated with a EEH, b TEX, c DPnB, and d EEA. The time dependencies of the emission maxima shown were fitted with exponential functions (solid lines). For bathochromic shift of these four formulations, one time constant was longer (17 to $31 \mathrm{~min})$ with a large contribution (4.1 to $4.7 \mathrm{~nm})$ while the other was shorter $(0.7$ to $1.5 \mathrm{~min}$ ) with a small contribution (1.5 to $2.9 \mathrm{~nm})$
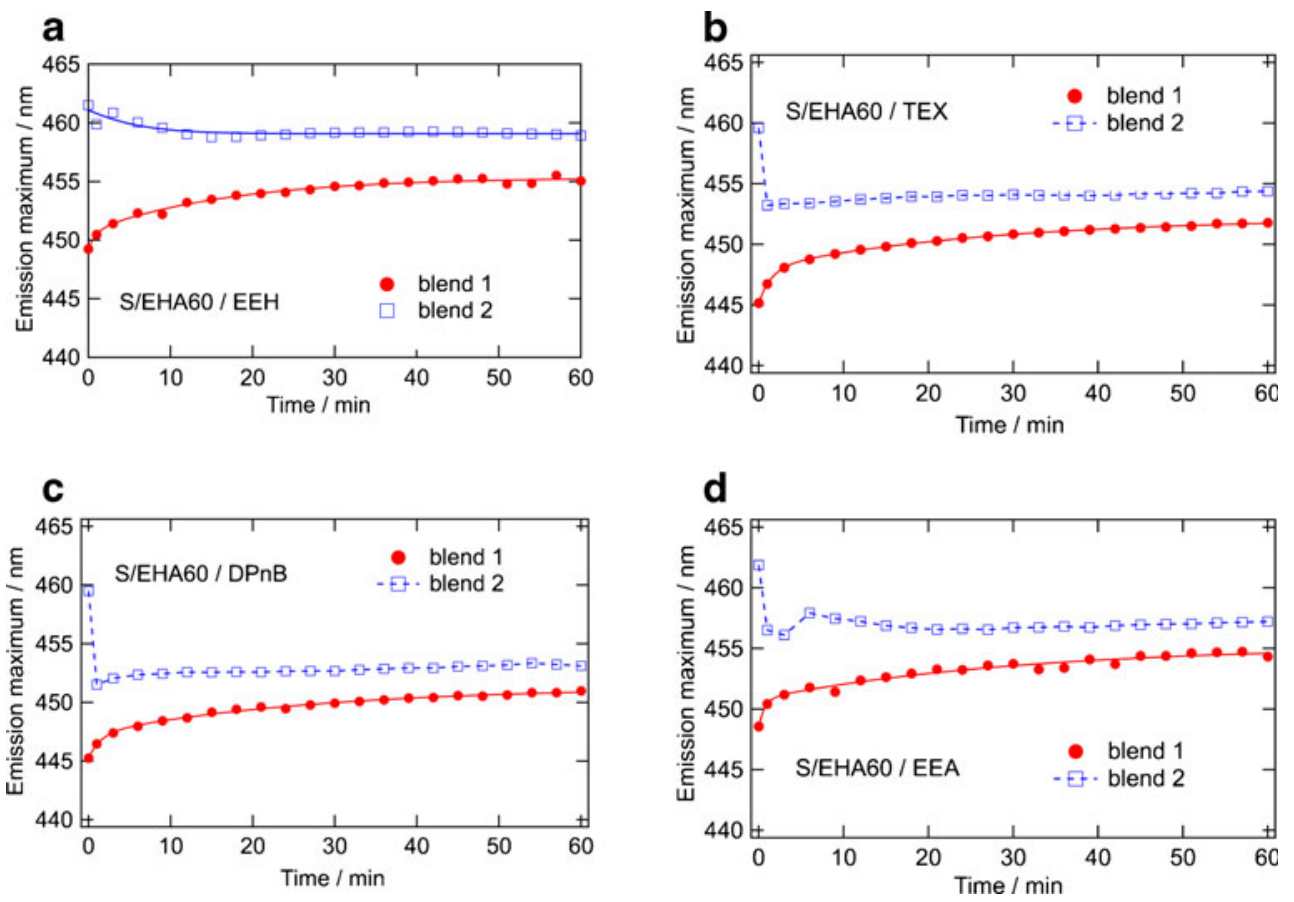
Table 4 Data obtained with (bi)exponential fitting of hypsochromic and bathochromic shifts in the emission maximum versus time plots of S/EHA $T_{\mathrm{g}} 60^{\circ} \mathrm{C}$ latex formulated with EEH, TEX, DPnB, and EEA

\begin{tabular}{lrrrr}
\hline $\begin{array}{l}\text { S/EHA } T_{\mathrm{g}} 60 \\
{ }^{\circ} \mathrm{C} \text { formulation }\end{array}$ & $\tau_{1}(\min )$ & $A_{1}(\mathrm{~nm})$ & $\tau_{2}(\min )$ & $A_{2}(\mathrm{~nm})$ \\
\hline EEH-hypsochromic & $6 \pm 1$ & $2.1 \pm 0.3$ & & \\
EEH-bathochromic & $17 \pm 2$ & $4.7 \pm 0.3$ & $0.9 \pm 0.3$ & $1.5 \pm 0.4$ \\
TEX-hypsochromic & Not fit & & & \\
TEX-bathochromic & $27 \pm 1$ & $4.15 \pm 0.05$ & $1.5 \pm 0.1$ & $2.9 \pm 0.1$ \\
DPnB-hypsochromic & Not fit & & & \\
DPnB-bathochromic & $27 \pm 3$ & $4.1 \pm 0.1$ & $1.5 \pm 0.2$ & $2.1 \pm 0.2$ \\
EEA-hypsochromic & Not fit & & & \\
EEA-bathochromic & $31 \pm 7$ & $4.4 \pm 0.4$ & $0.7 \pm 0.2$ & $2.3 \pm 0.4$ \\
\hline
\end{tabular}

Fits are shown in Fig. 8

both pairs of emulsions, and the actual kinetics could not be resolved. Diffusion of the co-solvent is fast when the free volume is high, which is the case at $T>T_{g}$ [18]. The polymer in the particles of the lower $T_{g}$ latices has a low viscosity, and addition of a co-solvent further reduces it. Hydroplasticization may contribute to a further lowering of the $T_{g}$ especially of the hydrophilic latex particles [32-34].

In the high $T_{g}$ latices, the emission maxima shifted more slowly, and the rates of the shift could be quantified in terms of exponential functions. A common observation was that the overall diffusion rate in blend 1 was slower than in blend 2 (Figs. 7 and 8): the hypsochromic shift, caused by the escape of co-solvent from the fluorescently labeled particles, occurred faster than the bathochromic shift due to the entry of co-solvent in the particles. In the first case, the latex particles had a low $T_{g}$ due to the presence of a co-solvent. Assuming that most of the co-solvent resides in the polymer particles, the $T_{g}$ of the solvent-swollen latex particles could be estimated according to the model of Toussaint et al. [8]. The predicted values were below ambient temperature, even for the high $T_{g}$ materials. The fluorescently labeled particles in blend 1 do not contain a co-solvent and have a higher $T_{g}$. In this case, however, plasticization by water still lowers the glass transition temperature compared to the dry state. Tsavalas and Sundberg recently proposed a method for estimating the effect of hydroplasticization on $T_{g}$ [34]. For the present cases, this predicts lowering of the $T_{g}$ by $6-7^{\circ}$ for the hydrophobic materials and $18-24^{\circ}$ for the hydrophilic ones. Thus, in the wet latex, the low $T_{g}$ materials are expected to have a $T_{g}$ below room temperature, but the high $T_{g}$ materials will still be in the glassy state under ambient conditions.

In the repartitioning of co-solvents, the molecules need to exit from the solvent-enriched polymer particles, enter into the water phase, diffuse to and enter into the co-solvent-deficient polymer particles. A simple description of the process in terms of a chemical equilibrium is given in Eq. 3:

$\mathrm{A} \underset{k_{\text {in }}}{\stackrel{k_{\text {out }}}{\rightleftarrows}} \mathrm{W} \underset{k_{\text {out }}}{\stackrel{k_{\text {in }}}{\rightleftarrows}} \mathrm{B}$

In this equation, $A$ and $B$ are the concentrations of the cosolvent in the two polymer compartments, $\mathrm{W}$ represents the concentration in the water phase. Since the polymer compartments are chemically identical, the same rate constants for entry $\left(k_{\text {in }}\right)$ and exit $\left(k_{\text {out }}\right)$ of the co-solvent will apply. Before blending, the co-solvent containing emulsions are in equilibrium. After blending equal volumes of $A+W$ and $W+$ $B$, the aqueous phase contains the same concentration of cosolvent as at the final equilibrium, but the concentration in compartment $A$ is twice the equilibrium value and that in $B$ is zero. If this simple model applies, the concentration in the aqueous phase is constant, that in $A$ decreases exponentially, and that in $B$ increases exponentially with the same time constant. The experimental data, however, clearly show different time constants and in many cases biphasic behavior.

Given the importance of the internal viscosity of the polymer particles, one should expect a rate that depends on the ability of plasticization by the co-solvent and its actual concentration in the particles. Because the concentration of the co-solvent is changing, we should expect the rate to change with time. This could lead to stretched exponential behavior [35]. The data, unfortunately, did not allow a meaningful fit to this type of function: many more data points are needed to determine the time constants and the stretch constants individually with a useful accuracy. We therefore used fits with a simple exponential or a biexponential function.

Even without any data fitting, it is obvious from Figs. 7 and 8 that the escape of co-solvent from the formulated particles is faster than the entrance into the non-formulated ones. Such behavior can be modeled by making $k_{\text {out }}$ dependent on the concentration of co-solvent in the particle. Initially, co-solvent escapes from the formulated particles, enriching the water phase in the co-solvent. In the next phase, the co-solvent in the water phase and the nonformulated particles slowly equilibrate. This is particularly clearly seen in the case of MMA/EA with EEA as the cosolvent: dilution of the formulated latex with the nonformulated one leads to rapid loss of co-solvent from the formulated particles ( $\tau=0.6 \mathrm{~min}$ ) and much slower increase of the co-solvent concentration in the non-formulated MFTlabeled particles ( $\tau=17 \mathrm{~min}$ ).

At this stage, we prefer to treat our observations more qualitatively. The migration of co-solvent out of the enriched particles will first occur from the outer shell, and likewise, entry into the non-formulated particles will first 
shift the emission of the probe's fluorescence in the outer shell. This may explain the rapid shift (with small amplitude) in the first minutes of most of the experiments with the high $T_{g}$ materials. As a result of this heterogeneity, the emission maximum as observed during the redistribution process does not necessarily agree precisely with the equilibrated values.

In the case of the high $T_{g} \mathrm{MMA} / \mathrm{EA}$ lattices, clear differences between the dynamics for the co-solvents are observed. For EEH equilibration was observed after $\sim 20 \mathrm{~min}$ of blend formation, whereas for DPnB and TEX, it took $\sim 40 \mathrm{~min}$. EEA took longer than the other three co-solvents, and equilibration was observed at $\sim 60 \mathrm{~min}$. This behavior correlates with the hydrophilicity of these co-solvents, which can be measured via the water solubility or the octanol/water partition coefficient $\left(\log \mathrm{P}_{\mathrm{o} / \mathrm{w}}\right)$. Texanol is the least water-soluble $(0.1 \%)$ and has the highest $\log \mathrm{P}_{\mathrm{o} / \mathrm{w}}(3.74)$ [36]. The water solubility of EEH is $0.2 \%$, and it has $\log \mathrm{P}_{\mathrm{o} / \mathrm{w}}=$ 2.73 [28]. DPnB being moderately water soluble (5\%) has a lower $\log \mathrm{P}_{\mathrm{o} / \mathrm{w}}=1.13$ [29]. EEA is quite water soluble (24\%) and has the lowest $\log \mathrm{P}_{\mathrm{o} / \mathrm{w}}=0.24$ [30]. Whereas release of cosolvent from the formulated particles is fast in all cases, the uptake in the non-formulated particles is faster when the water solubility is lower, that is, when the distribution coefficient favors the polymer phase more.

In Fig. 8, three features were observed in the plots for all four co-solvents with the high $T_{g}$ S/EHA latex: (1) escape of co-solvent from formulated fluorescently labeled particles was more rapid than the entrance, (2) in the bathochromic shift a fast rise occurred, and (3) coincidence of $\lambda_{\max }$ at a common equilibration point was not observed within $1 \mathrm{~h}$, although no more change in emission maximum was detected after $\sim 40 \mathrm{~min}$ of blend formation. The first observations are similar to those made with the hydrophilic latex materials. The fast rise of the bathochromic shift $(\tau, 0.7-$ $1.5 \mathrm{~min}$ ) can be attributed to the entry of the co-solvent in the outer shell of the particles, which is followed by slower equilibration $(\tau, 17-31 \mathrm{~min})$. This slow phase is slower than in the case of the MMA/EA latex, probably because the latter is much more plasticized by water. As mentioned above, the $T_{g}$ lowering by hydroplasticization in the high $T_{g}$ MMA/EA latex is estimated to be $\sim 24^{\circ}$, that for the $\mathrm{S} /$ EHA material only $\sim 7^{\circ}$. The third observation, the noncoincidence of the emission maxima after $1 \mathrm{~h}$, is unexpected. For most co-solvents, the hypsochromic shift is a bit smaller than the bathochromic one. It is conceivable that the presence of a high concentration of the co-solvent in the latex $(9 \% w / w$ on the total weight, i.e., up to $24 \%$ in the polymer phase) causes a structural relaxation of the polymer particles. As a result, the two polymer compartments $A$ and $B$ (Eq. 3) are no longer identical on the time scale of the experiment. The relaxation of compartment $A$ may have increased its affinity for the co-solvent, or it may have increased the sensitivity of the solvatochromic fluorescent probe to the presence of the co-solvent. It is also possible, however, that there is a minor very slow phase in the repartitioning process. Further research will be needed to shed more light on this issue.

\section{Conclusion}

Repartitioning of co-solvents was monitored as a function of time in low and high $T_{g}$ hydrophobic S/EHA and hydrophilic MMA/EA acrylic latices by means of a solvatochromic fluorescent probe. Low $T_{g}$ latices of both types displayed rapid repartitioning of co-solvent across the aqueous and polymer phases of the latex, and equilibration was observed within the first minute of blend formation.

In high $T_{g} \mathrm{MMA} / \mathrm{EA}$ latex, repartitioning of cosolvent smoothly progressed towards equilibration, which was achieved within an hour of blending. The equilibrium was attained faster with decreasing water solubility of the co-solvents. In high $T_{g}$ S/EHA latex, repartitioning of co-solvent was slower than in the MMA/EA latex of similar (dry state) $T_{g}$. This may be due to a lower wet state $T_{g}$ in the latter as a result of hydroplasticization.

Unexpectedly, a common equilibration point was not reached in the complementary experiments starting with the co-solvent in the labeled or in the non-labeled S/EHA latex. A possible explanation is that the presence of a high concentration of the co-solvent in the latex causes a change of the material that is not reversible on the time scale of the present experiments. A minor slow phase in the repartitioning cannot be excluded, however.

Biexponential fits provided two time constants for the entry of the co-solvent in the non-formulated particles in both high $T_{g}$ latices, which demonstrate that diffusion occurs in two different stages: a fast component with small amplitude, which is particularly pronounced in the case of the hydrophobic material, is probably caused by diffusion of co-solvent molecules into the outer shells of the latex particles, and the second, slower component is related to further penetration of co-solvent molecules into the cores of the particles.

Acknowledgments This research is part of the Dutch Polymer Institute (DPI) (project number 606). We thank Koen Biemans (DSM) for his help with the polymer synthesis and characterization.

Open Access This article is distributed under the terms of the Creative Commons Attribution Noncommercial License which permits any noncommercial use, distribution, and reproduction in any medium, provided the original author(s) and source are credited. 


\section{References}

1. Padget JC (1994) Polymers for water-based coatings - a systematic overview. J Coatings Technol 66(839):89-105

2. Athawale VD, Nimbalkar RV (2011) Waterborne coatings based on renewable oil resources: an overview. J Am Oil Chem Soc 88 (2):159-185. doi:10.1007/s11746-010-1668-9

3. Overbeek A (2011) Polymer heterogeneity in waterborne coatings. J Coatings Technol Res 7(1):1-21. doi:10.1007/s11998-009-9201-5

4. Keddie JL (1997) Film formation of latex. Mat Sci Eng R 21 (3):101-170

5. Winnik MA (2002) Interdiffusion and crosslinking in thermoset latex films. J Coatings Technol 74(925):49-63

6. van Herk AM (2005) Chemistry and technology of emulsion polymerization. Blackwell, Oxford

7. Steward PA, Hearn J, Wilkinson MC (2000) An overview of polymer latex film formation and properties. Adv Colloid Interface Sci 86(3):195-267

8. Toussaint A, DeWilde M, Molenaar F, Mulvihill J (1997) Calculation of Tg and MFFT depression due to added coalescing agents. Prog Org Coat 30(3):179-184

9. Keddie JL, Routh AF (2010) Fundamentals of latex film formation: process and properties. Springer, Dordrecht

10. Wall FT (1950) Principles of emulsion polymerization. Science 111:82-84

11. Toussaint A, DeWilde M (1997) A method to predict the distribution coefficient of coalescing agents between latex particles and the water phase. Prog Org Coat 30(3):173-177

12. Ranjbar Z, Rastegar S (2006) Influence of co-solvent content on electro-deposition behavior of acrylic lattices of different glass transition temperatures. Prog Org Coat 57(4):365-370. doi:10.1016/j.porgcoat.2006.09.015

13. Zohrehvand S, te Nijenhuis K (2005) Film formation from monodisperse acrylic latices, part 4: the role of coalescing agents in the film formation process. Colloid Polym Sci 283(12):1305-1312. doi:10.1007/s00396-005-1333-2

14. te Nijenhuis K, Zohrehvand S (2005) Film formation from monodisperse acrylic latices 2 . Influence of drying temperature on the film formation process. J Colloid Interface Sci 284(1):129-138. doi:10.1016/j.jcis.2004.09.077

15. Zohrehvand S, te Nijenhuis K (2005) Film formation from monodisperse acrylic latices-5. Drying and ageing in coalescing agent containing latex films. J Colloid Interface Sci 288(1):75-82. doi:10.1016/j.jcis.2005.02.059

16. Lahtinen M, Glad E, Koskimies S, Sundholm F, Rissanen K (2003) Synthesis of novel reactive coalescing agents and their application in a latex coating. J Appl Polym Sci 87(4):610-615. doi:10.1002/ app. 11336

17. Seebergh JE, Berg JC (1997) The effect of organic cosolvent on the aggregation stability of an aqueous polystyrene latex dispersion. Colloids and Surfaces a-Physicochem and Eng Asp 121(1):89-98

18. Fu Z, Hejl A, Swartz A (2009) Polymer mixing enhances performance. Eur Coat J 6:26-33

19. Vignola E, Wood KA, Hedhli L (2003) Electrophoretic mobility evidence of surfactant partitioning in a waterborne latex. Colloid Polym Sci 282(1):64-68. doi:10.1007/s00396-003-0918-x

20. Schroeder WF, Liu YQ, Tomba JP, Soleimani M, Lau W, Winnik MA (2010) Influence of ethylene glycol and propylene glycol on polymer diffusion in poly(butyl acrylate-co-methyl methacrylate) latex films. J Phys Chem B 114(9):3085-3094. doi:10.1021/jp9118875

21. Juhué D, Lang J (1994) Latex film formation in the presence of organic solvents. Macromolecules 27(3):695-701

22. Juhué D, Wang YC, Winnik MA, Haley F (1993) Influence of a coalescing aid on polymer diffusion in poly(butyl methacrylate) latex films. Makromol Chem Rapid 14(6):345-349

23. Brouwer AM, Raja TN, Biemans K, Nabuurs T, Tennebroek R (2008) Probing of cosolvents in polymer latex materials by using solvatochromic fluorescence. Ann N Y Acad Sci. doi:10.1196/ annals. 1430.014

24. Raja TN, Brouwer AM, Biemans K, Nabuurs T, Tennebroek R (2010) Detection of coalescing agents in water borne latex emulsions using an environment sensitive fluorescent probe. Photochem Photobiol Sci 9:975-984. doi:10.1039/C0PP00065E

25. Goes M, Lauteslager XY, Verhoeven JW, Hofstraat JW (1998) A blue excitable charge-transfer fluorescent probe and its fluorogenic derivative. Eur J Org Chem 11:2373-2377

26. Fox TG, Flory PJ (1950) Second-order transition temperatures and related propertoes of polystyrene. I. Influence of molecular weight. J Appl Phys 21:581-591

27. Eastman Chemical Company (2004) Texanol ester alcohol: the coalescing aid for latex paints. Publication M-205F. Eastman Chemical Company, Kingsport

28. National Toxicology Program (2008) Chemical information profile for ethylene glycol 2-ethylhexyl ether (EEH). National Toxicology Program, Durham

29. Dow Chemical Company Ltd (2007) Material safety data sheet (MSDS) of dipropylene glycol n-butylether (DPnB). Dow Chemical Company Ltd., Cambridge

30. Caledon Laboratories Ltd (2010) Material safety data sheet (MSDS) of ethylene glycol monoethyl ether acetate (EEA). Caledon Laboratories Ltd., Ontario

31. Lauteslager XY, van Stokkum IHM, van Ramesdonk HJ, Brouwer AM, Verhoeven JW (1999) Conformational dynamics of semiflexibly bridged donor-acceptor systems studied with a streak camera and spectrotemporal parametrization of fluorescence. J Phys Chem A 103(6):653-659

32. Soleimani M, Haley JC, Lau W, Winnik MA (2009) Effect of hydroplasticization on polymer diffusion in poly(butyl acrylateco-methyl methacrylate) and poly(2-ethylhexyl acrylate-co-tertbutyl methacrylate) latex films. Macromolecules 43:975-985. doi:10.1021/ma9020483

33. Jiang B, Tsavalas J, Sundberg D (2010) Measuring the glass transition of latex-based polymers in the hydroplasticized state via differential scanning calorimetry. Langmuir 26(12):94089415. doi:10.1021/la100425g

34. Tsavalas JG, Sundberg DC (2010) Hydroplasticization of polymers: model predictions and application to emulsion polymers. Langmuir 26(10):6960-6966. doi:10.1021/la904211e

35. Lee KCB, Siegel J, Webb SED, Leveque-Fort S, Cole MJ, Jones R, Dowling K, Lever MJ, French PMW (2001) Application of the stretched exponential function to fluorescence lifetime imaging. Biophys J 81(3):1265-1274

36. High Production Volume Chemicals Programme (1994) Screening information data sheet (SIDS) of 2,2,4-trimethyl-1,3-pentanediol monoisobutyrate (Texanol). Organisation for Economic $\mathrm{Co}$ operation and Development (OECD). High Production Volume Chemicals Programme, USA 